\title{
3D Reconstruction of Surfaces with Steep Slopes from Multiple SEM Images
}

\author{
R. Danzl*, F. Helmli* and S. Scherer* \\ *Alicona Imaging GmbH, Teslastraße 8, A-8074 Grambach, Austria,
}

The three-dimensional measurement of microscopic surfaces with steep slopes is a big challenge for many measurement devices. Although the scanning electron microscope (SEM) offers a large depth of focus and high contrast images with lateral resolutions up to $1 \mathrm{~nm}$ it only delivers twodimensional images of three-dimensional objects. In [1][2], however, a 3D surface reconstruction method has been proposed that relies on the triangulation principle and exploits stereoscopic information inherent in two or three images obtained from the SEM (scanning electron microscope) by tilting a probe using a eucentric stage by several degrees. We propose an improved version of $[1][2]$ which can be used to reconstruct even very steep surface slopes.

If only 2 input images are available important calibration parameters such as the tilt angle between the images have to be set by the operator. From many tilt stages the angle can be read only with an accuracy of about $0.5^{\circ}$ which has a strong influence on the accuracy of the results [1]. A method that operates on three input images [2] allows to automatically estimate the true tilt angle up to an accuracy of $0.1^{\circ}$, thus leading to a significantly increased accuracy of height measurements.

In this paper we propose an extension to [2] which uses a new and robust version for calibration data refinement that solves for all orientation parameters of the input images (instead of the main tilt angles only). This allows the use of a new sophisticated 3D reconstruction method which does not only search for corresponding features in two images but in all three images at the same time. This approach improves the reconstruction of small features, of objects with repeating structures and of objects that would be occluded if only two images were present.

Especially the reconstruction of surfaces with steep slopes can be improved by using multiple images. Fig. 1a shows an input image obtained by the SEM from a small region of a micro-contour artefact from the PTB (Physikalisch Technische Bundesanstalt, Braunschweig, Germany). The region shows a geometric object with steep slopes having nominal angles of $80^{\circ}$. In Fig. 1c a 3D model of the surface is provided which has been reconstructed using the new algorithm which is included in the MeX software by Alicona Imaging. Fig. 1d shows the same model in pseudo color mode where each color represents a specific height. In order to measure the slope of the surface, a profile has been extracted along a specified path (Fig 1b). The angle between the flanks has been measured by fitting two lines into the profile. The resulting angle of $19.59^{\circ}$ is very close to the calibrated value of $19.54^{\circ}$ from the PTB. The crossing point of the two fitted lines is $803.75 \mu \mathrm{m}$, the calibrated value is $803 \mu \mathrm{m}$, showing the accuracy of the proposed method.

\section{References}

[1] S. Scherer. "3D Surface analysis in Scanning electron Microscopy". G.I.T. Imaging \& Microscopy 3 ( 2002) 45-46.

[2] H. Schröttner, M. Schmied and S. Scherer. "Robust, dense and accurate 3D surface reconstruction in SEM through automatic calibration data calculation from multiple images". Proceedings EMC Vol 1. (2004). Antwerp, Belgium, 441-442. 


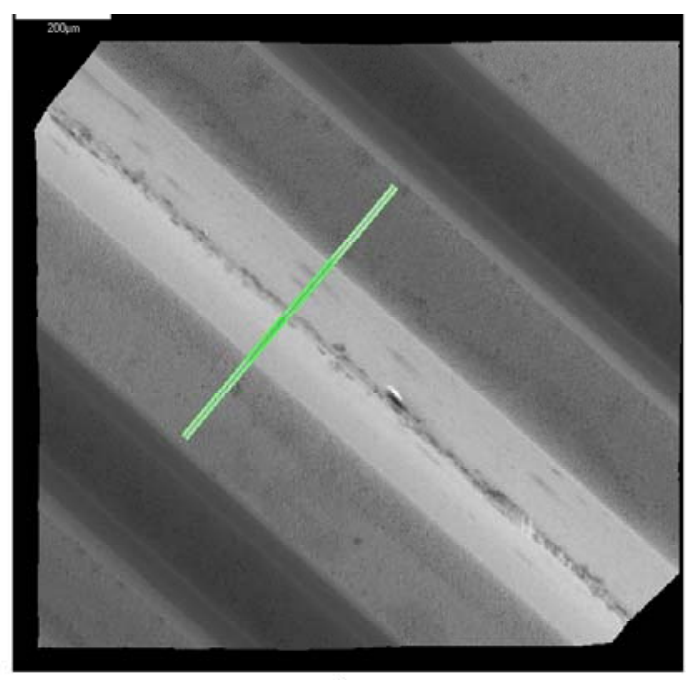

a)
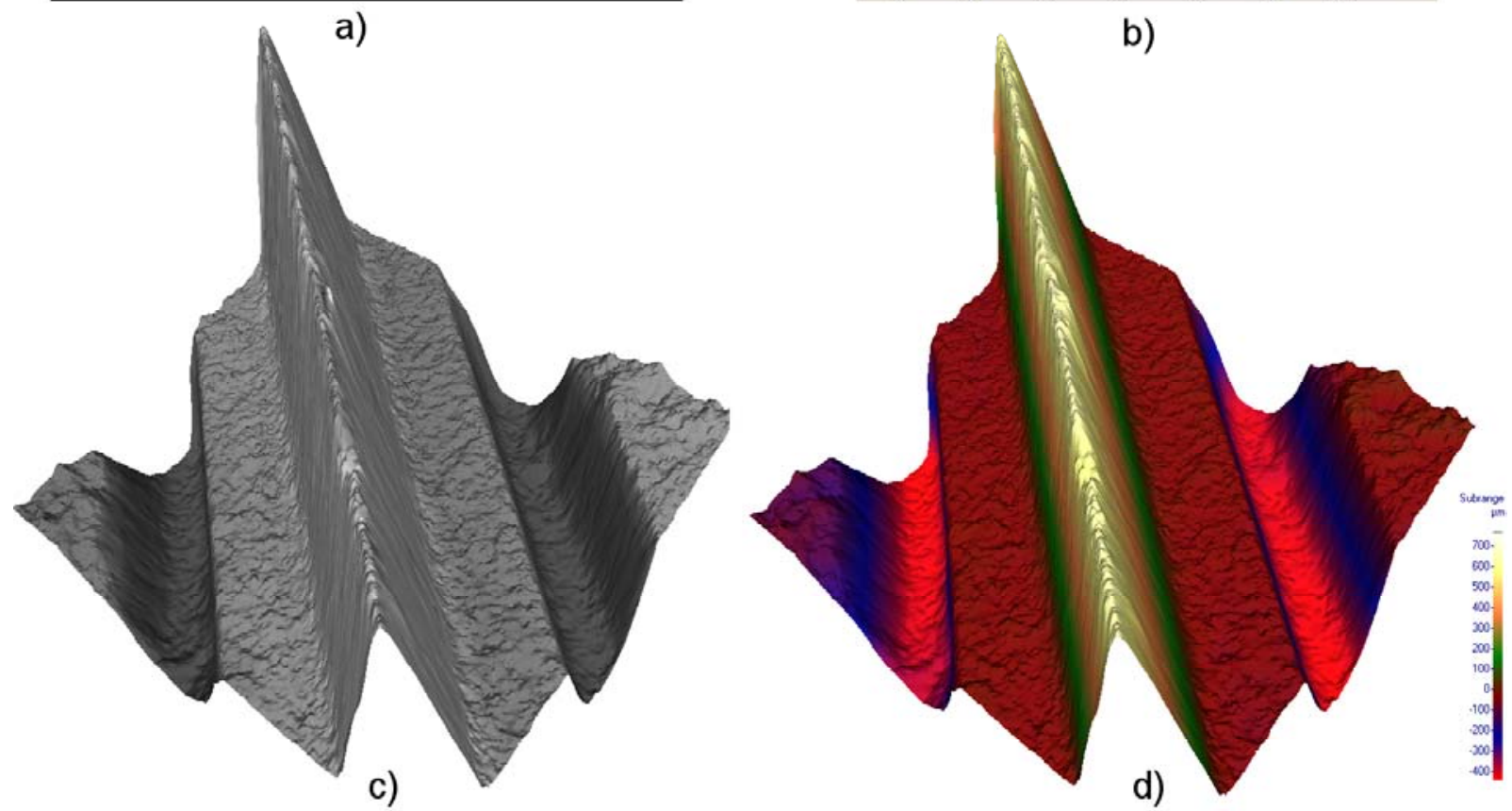

Fig. 1. a): One of the three input images from the SEM with the profile path used in Fig. 1b. b): Angle measurement from an extracted surface profile. c): 3D model obtained using the new algorithm. D): 3D model in pseudo-color mode. 